\title{
Low Current Collapse and Low Leakage GaN MIS-HEMT Using AIN/SiN as Gate Dielectric and Passivation Layer
}

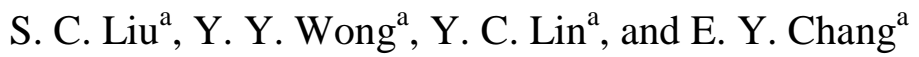 \\ a Department of Materials Science and Engineering, National Chiao-Tung University, \\ Hsinchu, 300 Taiwan.
}

\begin{abstract}
An effective passivation and gate insulator with low current collapse and improved dynamic ON-state resistance $\left(R_{\mathrm{ON}}\right)$ for $\mathrm{GaN}$ MIS-HEMT is demonstrated in this work. The structure of passivation and gate insulator is fabricated by 4-nm $\mathrm{SiN}$ as the first passivation layer and 1-nm AlN. The bilayer AlN/SiN structure integrates the advantages of $\mathrm{SiN}$ and $\mathrm{AlN}$. SiN passivation has been proved to effectively reduce GaN surface states. AlN has high bandgap of $\sim 6.2 \mathrm{eV}$ which can suppress leakage current. Hence, the unfavorable effects such as trapping effect and leakage current which will induce current collapse and are effectively suppressed by using AlN/SiN bilayer thin film. A GaN MIS-HEMT with AlN/SiN passivation and gate dielectric exhibits improved $I-V$ characteristics, low leakage current, low current collapse, and improved dynamic $R_{\mathrm{ON}}$ at high quiescent drain bias of $100 \mathrm{~V}$.
\end{abstract}

\section{Introduction}

Gallium nitride-based high-electron-mobility transistors (GaN HEMTs) have demonstrated outstanding performance for high-power and high-frequency applications for defense and communication systems. However, there are many undesirable effects such as current collapse and increase in dynamic ON-resistance $\left(R_{\mathrm{ON}, \mathrm{DYN}}\right)$ due to the surface states and the high polarization nature of the material [1], [2]. Passivation proved to reduce surface states and surface potential can effectively mitigate current collapse and increase of $R_{\mathrm{ON}, \mathrm{DYN}}$. Different dielectric layers such as $\mathrm{SiN}, \mathrm{SiO}$ [3], and $\mathrm{Al}_{2} \mathrm{O}_{3}$ [4] have been used as the gate dielectric layer and/or the passivation layer for GaN metalinsulator-semiconductor HEMTs (MIS-HEMTs). Nitride-based materials are more desirable for $\mathrm{GaN}$ passivation because the oxygen impurities can easily diffuse into $\mathrm{GaN}$ structure inducing deep levels traps. SiN has been proved as an effective passivation dielectric to reduce the surface states and can efficiently suppress current collapse in the GaN HEMTs [5]. However, the bandgap of $\mathrm{SiN}(\sim 5 \mathrm{eV})$ which is little higher than AlGaN $(\sim 4 \mathrm{eV})$ is not high enough to suppress leakage current. AlN with a large bandgap $(\sim 6.2 \mathrm{eV})$ was proved to effectively reduce leakage current as gate dielectric and passivation layer [6]. In this work, we demonstrate GaN MIS-HEMT with improvements in current voltage characteristics, current collapse, and dynamic ON-resistance utilizing AlN/SiN bilayer gate dielectric and passivation layer which combine the advantages of $\mathrm{SiN}$ and AlN.

\section{Device Fabrication}

The AlGaN/GaN HEMT heterostructure was grown by metal-organic chemical vapor deposition (MOCVD) on silicon substrate with $\sim 1 \mathrm{~nm}$ heavily doped GaN on the top to 
form low contact resistance ohmic contacts. The wafer was divided into three samples after mesa and ohmic contact process. Then the SiN thin film was deposited by PECVD and the AlN thin film was deposited by plasma-enhanced atomic layer deposition (PEALD). Before thin film deposition, nitrogen plasma was adopted for cleaning GaN surface, then thin film was deposited in situ after nitrogen plasma treatment. For comparing the performance of GaN MIS-HEMT with AlN/SiN bilayer gate dielectric, we also prepared reference devices with single layers dielectric. The dielectric and passivation layer were prepared differently for each sample: sample A with 5-nm SiN, sample B with 1-nm AlN/4-nm SiN, sample C with 5-nm AlN. The gate-to-drain spacing $L_{\mathrm{GD}}$, gate-to-source spacing $L_{\mathrm{GS}}$, and gate length $L_{\mathrm{G}}$ were $4 \mu \mathrm{m}, 2 \mu \mathrm{m}$, and $1 \mu \mathrm{m}$, respectively.

\section{Results and Discussion}

Fig. 1 shows the $I_{\mathrm{D}}-V_{\mathrm{D}}$ characteristics, where the drain current curves were measured at $V_{\mathrm{G}}=0 \mathrm{~V}$. The SiN first passivated devices have higher $I_{\mathrm{D}}$ and lower $R_{\mathrm{ON}}$ as compared to AlN first passivated device. The $R_{\mathrm{ON}}$ were $0.3 \mathrm{~m} \Omega \cdot \mathrm{cm}^{2}, 0.3 \mathrm{~m} \Omega \cdot \mathrm{cm}^{2}$, and $0.33 \mathrm{~m} \Omega \cdot$ $\mathrm{cm}^{2}$ for sample A, sample B, and sample C, respectively. For gate leakage shown in Fig. 2, GaN MIS-HEMTs using AlN as gate dielectric have lower gate leakage current. It suggests AlN with high bandgap can effectively suppress leakage current.

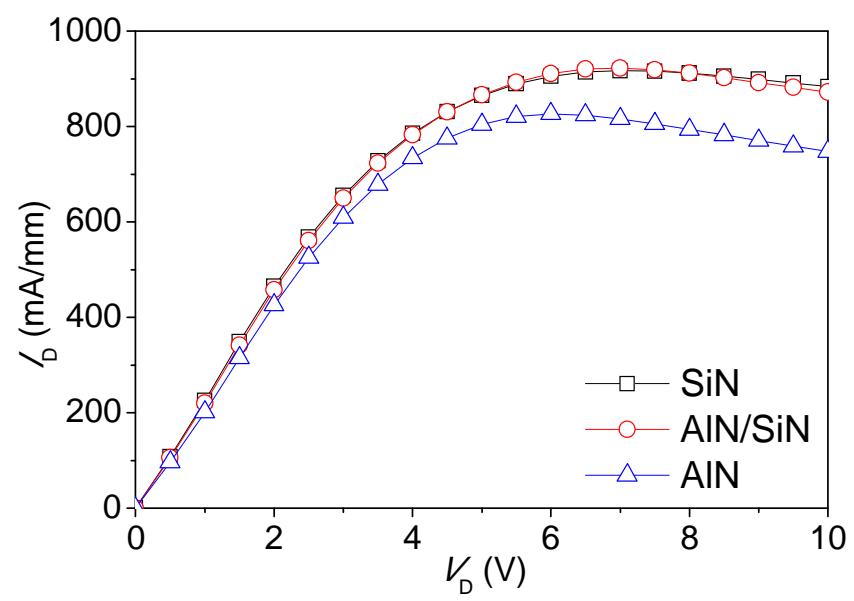

Fig. $1 I_{\mathrm{D}}-V_{\mathrm{D}}$ characteristics of GaN HEMTs with different surface passivation layers.

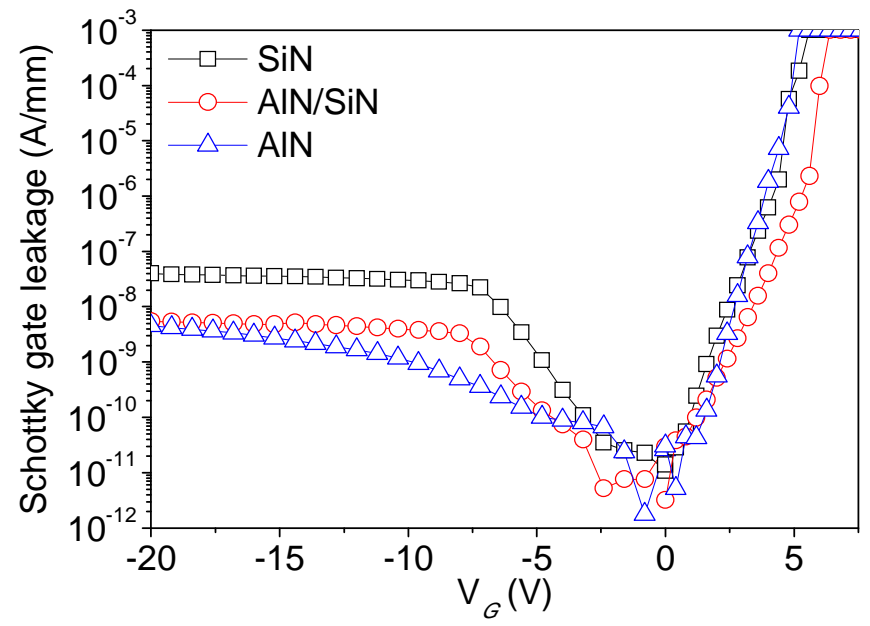

Fig. 2 Gate leakage current comparison in MIS-HEMTs structure. 
Fig. 3 shows the percent degradation of $R_{\mathrm{ON}, \mathrm{DYN}}$ versus time. The OFF-state quiescent stress bias were set at $V_{\mathrm{DSQ}}=100 \mathrm{~V}$ and $V_{\mathrm{GSQ}}=-10 \mathrm{~V}$. With the high-drain-bias stress and OFF-state stress, electrons can easily go through gate dielectric from gate then be trapped into interface state and GaN structure. These trapped electrons can deplete 2DEG channel while GaN HEMTs fast switch from OFF-state to ON-state, causing current collapse and increase of dynamic ON-state resistance. The ON-state $R_{\mathrm{ON}, \mathrm{DYN}}$ is extracted at a gate voltage of $0 \mathrm{~V}$ and a drain voltage of $2 \mathrm{~V}$. $R_{\mathrm{ON}, \mathrm{DYN}}$ degradation reflects the passivation effects on GaN after stress. GaN MIS-HEMT with AIN/SiN obviously suppresses $R_{\mathrm{ON} \text {,DYN }}$ degradation compared to the single layer samples. Also, the SiN first passivated devices have slight $R_{\mathrm{ON}, \mathrm{DYN}}$ degradation. It indicates $\mathrm{SiN}$ has low interface trapping density on GaN surface and AlN can reduce trapping electrons charged through gate leakage. In conclusion, $\mathrm{SiN}$ was been proved that it has many good effects for $\mathrm{GaN}$ passivation such as decreasing of channel resistance, low surface state, low interface trapping density and low current collapse effect. AlN with high bandgap nature can suppress the leakage current. However, it would increase channel resistance, and cause severe current collapse effect. In this study, an effective AlN/SiN bilayer dielectric and passivation layer have been demonstrated for reducing current collapse effect and leakage current in GaN MIS-HEMT.

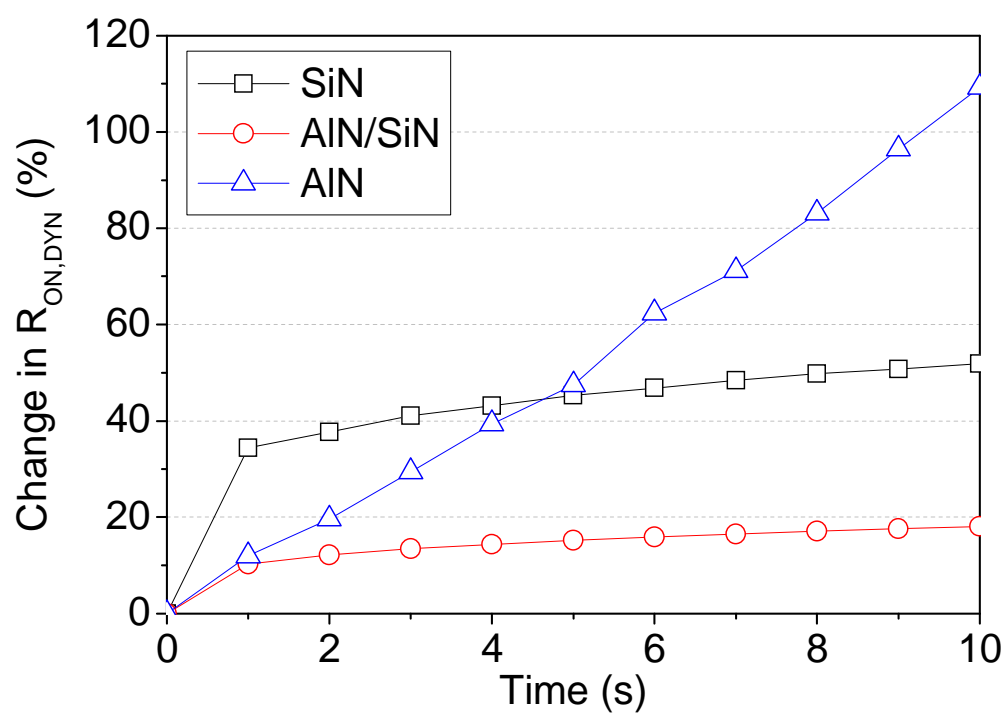

Fig. 3 Percent degradation of $R_{\mathrm{ON}, \mathrm{DYN}}$ versus time.

\section{Conclusion}

A GaN MIS-HEMT with AlN/SiN bilayer passivation and gate dielectric consists of high bandgap and high quality $\mathrm{SiN} / \mathrm{GaN}$ interface. It demonstrates improved $I-V$ characteristics, low leakage current, low current collapse, and improved dynamic $R_{\mathrm{ON}}$. Comparing to the single layer samples, the change in dynamic $R_{\mathrm{ON}}$ of AlN/SiN bilayer sample is much lower while devices were operating at high quiescent drain bias.

\section{Acknowledgments}

The author would like to acknowledge all colleagues in this work. This work was supported in part by National Nano Device Laboratories and National Chiao Tung University Nano Facility Center. 


\section{References}

1. S. Binari, K. Ikossi, J. A. Rousos, et al., IEEE Trans. Electron Devices, vol. 48, no. 3, pp. 465-471, (2001).

2. D. Jin and J. A. Alamo, IEEE Trans. Electron Devices, vol. 60, no. 10, pp. 31903196, (2013).

3. S. Arulkumaran, T. Egawa, H. Ishikawa, T. Jimbo, and Y. Sano, Appl. Phys. Lett., Vol. 84, No. 4, (2004).

4. T. Hashizume, S. Ootomo, and H. Hasegawa, Appl. Phys. Lett., Vol. 83, No. 14, (2003).

5. B. Green and K. Chu, IEEE Electron Device Lett., vol. 21, no. 6, pp. 268-270, (2000).

6. Z. Tang, S. Huang, Q. Jiang, S. Liu, C. Liu, and K. J. Chen, IEEE Electron Device Lett., vol. 34, no. 3, pp. 366-368, (2013). 